\title{
Avaliação da ingestão de leite e substitutos e do hábito do desjejum de universitários do curso de nutrição
}

\author{
Loraine Gollino ${ }^{1}$, Ana Carolina Campi Cansian ${ }^{1}$, Joana Bevilacqua de Oliveira Alves ${ }^{1}$, Estefânia \\ Maria Soares Pereira ${ }^{2}$
}

A pesquisa visou avaliar a ingestão de leite e substitutos, o hábito do desjejum e a percepção qualitativa do desjejum após o ingresso na instituição dos estudantes universitários de Nutrição da Universidade Federal do Triângulo Mineiro (UFTM), Uberaba (Minas Gerais). A amostra foi constituída por 111 estudantes, do primeiro ao oitavo semestre, de ambos os gêneros, com média de idade de 21 anos. A análise da ingestão alimentar foi feita pelo Questionário Semi-Quantitativo de Frequência Alimentar (QSQF) e perguntas sobre o hábito e consumo do desjejum. Os resultados mostraram que 37\% dos estudantes ingeriam de 3 ou mais porções de leite e/ou substitutos diariamente e em relação ao hábito do desjejum, (60,36\%) dos estudantes realizavam todos os dias. $\mathrm{Na}$ auto-avaliação do desjejum 46\% classificaram como "bom" e 34,2\% como "regular" e quanto as modificações alimentares ao ingressar na Universidade, 53,1\% identificaram que houve mudança e, dentre estes, $54,2 \%$ classificaram como benéfica. Nesta pesquisa foram identificados bons hábitos entre os estudantes. Análises que envolvam os estudantes, vinculados à informação nutricional, tornam-se valiosas por fazerem parte da classe de futuros multiplicadores da área da saúde.

Palavras-chave: estudantes universitários, hábito do desjejum, leite e substitutos.

\section{Assessment on milk intake and its substitutes: eating habits in breakfast of College students majoring in Nutrition}

The research aimed to evaluate the ingestion of milk and its substitutes, eating habits in breakfast, and the qualitative perception of students after their admission in the Nutrition Course at the Federal University of Triangulo Mineiro (UFTM), Uberaba (Minas Gerais). The sample consisted of 111 students from freshman to senior years, both genders, with an average age of 21. The diet ingest analysis was done by the Feed Frequency SemiQuantitative Questionnaire (acronym in Portuguese, QSQF) and also by open questions regarding breakfast habits and consumption. The results showed that $37 \%$ of the students ate up to 3 or more portions of milk and $\backslash$ or substitutes daily. In regard of breakfast habits, a great number of students $(60.36 \%)$ specified their diet for daily breakfast. In the breakfast self-assessment $46 \%$ of the students classified as "good" and $34.2 \%$ as "regular". For dietary changes after entering university, 53.1\% of the students identified changes in their eating habits, in which $54.2 \%$ among them classified such changes as beneficial. In this research, there was a positive factor related to eating habits among college students. Analysis involving students with nutritional information, become valuable once they will contribute with their knowledge in the health field.

Key-words: college students, milk intake, breakfast habits.

\footnotetext{
${ }^{1}$ Departamento de Clínica Médica (UNESP-FMB). Correspondência: Avenida Sete, no 953, CEP: 14620-000, Orlândia - SP. Tel: (16) 98110-4477. E-mail: lo_gollino@hotmail.com.

${ }^{2}$ Docente e Coordenadora do curso da Graduação em Nutrição na Universidade Federal do Triângulo Mineiro - UFTM. 


\section{INTRODUÇÃO}

A vida universitária pode ser considerada o início de mudanças, principalmente aquelas relacionadas com a alimentação. Os estudantes vivem novas experiências ao deixarem a casa dos pais, aumentando a responsabilidade pela própria vida e pela preparação das refeições ${ }^{[1,2]}$. A universidade enquanto instituição tem importante papel como formadora de hábitos ${ }^{[1]}$.

Em estudo conduzido por Vieira [3], a preocupação com a saúde foi encontrada em $40 \%$ dos adolescentes, apesar do desequilíbrio em suas dietas, principalmente, pela omissão de alguma refeição (desjejum, almoço e jantar). Neste mesmo estudo, 50\% dos estudantes entrevistados relataram ingerir maior quantidade de alimentos após o ingresso na universidade.

O consumo de nutrientes deve ser avaliado de forma qualitativa e quantitativa pelo comportamento alimentar, desta forma, colaborando para melhorias na qualidade de vida [4].

As principais fontes alimentares de cálcio são o leite e seus substitutos, queijo e iogurte, fornecendo além do mineral cálcio que é nutriente essencial, necessário em diversas funções biológicas, outros nutrientes importantes para o organismo, como a proteína, potássio, fósforo [5]. Para atingir a recomendação diária de cálcio é preciso consumir 3 porções de leite de $250 \mathrm{~mL}$, o qual cada porção pode ser substituída por 3 fatias de queijo mussarela, 1 pote de $140 \mathrm{~g}$ de iogurte de frutas e 1 colher e meia de sopa de requeijão cremoso [6].

Por meio do estudo conduzido por Porto [7], foi verificado reduzido consumo de leite e substitutos para atingir as recomendações diárias de cálcio de 1.300 mg em escolares de 11 a 18 anos. Nesse estudo, apenas 3 das 142 estudantes do sexo feminino atingiram a cota diária preconizada de cálcio e a média de ingestão da população do estudo foi de apenas $441,6 \mathrm{mg} / \mathrm{dia}$.

Estudo realizado em quatro municípios brasileiros (Rio de Janeiro, Goiânia, Ouro Preto e Campinas) verificou a inadequação da disponibilidade do cálcio em relação à faixa de renda familiar, demonstrando que com a menor renda familiar há o elevado risco para não atingir as recomendações de cálcio necessárias [8].

A ingestão adequada de cálcio é necessária para permitir ganhos do mineral na densidade e formação óssea, possibilitando fatores positivos na prevenção da osteoporose, também é necessário para a transmissão nervosa e regulação da função muscular [?].

O desjejum é a primeira refeição do dia, geralmente após o jejum de mais de doze horas, associando-se a um hábito alimentar adequado com promoção de mudanças no desenvolvimento cognitivo, no comportamento e na memória, fornecendo nutrientes essenciais ao organismo para o seu funcionamento, sendo uma das refeições mais importantes do dia [10]. Durante os últimos 30 anos houve uma redução no consumo do desjejum [2], sendo considerada a refeição mais negligenciada pelos adolescentes [11].

Com a submissão a um jejum superior a dez horas existem problemas quanto ao raciocínio lógico, à atenção e em relação a fases do funcionamento cognitivo, devido, provavelmente, a redução da síntese de neurotransmissores influenciada pela ausência de substrato proteico e baixas concentrações de glicose no cérebro. Para estabelecer um bom rendimento intelectual do período da manhã é de extrema importância realizar a primeira refeição, sendo adequada do ponto de vista nutricional [12,13].

A omissão ou o desjejum inadequado do ponto de vista nutricional influencia o aumento do nível da fome durante o dia [2]. $\mathrm{O}$ ato de omitir o desjejum relaciona-se no consumo reduzido de alimentos ricos em cálcio e vitamina $\mathrm{D}$, uma vez que os alimentos fonte de cálcio e de vitamina $\mathrm{D}$ são os mais consumidos no desjejum [11,14].

Entre os alimentos consumidos no desjejum mais relatados em vários estudos foram o pão, o leite com achocolatado, com café e com frutas, o queijo, a margarina, o açúcar e as frutas ou sucos [11,15,10,16].

Estudantes adolescentes que consomem um desjejum de boa qualidade possuem, geralmente, um melhor padrão dietético em relação àqueles que consomem um desjejum de baixa qualidade [17]. 
O hábito alimentar se expressa na relação cultural e social de cada pessoa estabelecida com o meio em que vive e este hábito individual reflete nos valores da sociedade. No estudo de Vieira et al. [3] foi verificado que $60 \%$ dos entrevistados relataram omitir uma das refeições principais (almoço, jantar, desjejum), sendo que $37 \%$ não realizavam o desjejum. Marcondelli et al. [18] encontraram 79,7\% dos entrevistados com inadequação do hábito alimentar e os menores percentuais foram para o consumo de leite e derivados, com 23,1\%. A eficácia no aumento da ingestão de alimentos fontes de cálcio entre os adolescentes está relacionada com o ambiente familiar ${ }^{[19]}$.

Outro estudo verificou que dos adolescentes entrevistados, 63,6\% não realizavam o desjejum e a colação [20]; $56 \%$ não realizavam o desjejum, a ceia ou colação [11]; $60 \%$ não tinham o hábito de realizar as três refeições consideradas principais e a omissão do desjejum ocorria em 37\% dos estudantes [3]; 30,5\% realizavam o desjejum diariamente e os estudantes que cursavam a graduação em nutrição, em função do aprendizado proporcionado pelo curso, relatavam a maior alteração positiva quanto ao consumo do desjejum, entre outros cursos ${ }^{[10]}$.

A relação entre o consumo de produtos lácteos, a realização do desjejum e a comparação entre gêneros foram relatados em estudos que mostraram diferenças estatisticamente significativas, em que a ingestão de produtos lácteos e a realização do desjejum padrão, contendo alimentos fonte de cálcio e de energia prevaleceram entre os meninos [11,21,22,19].

Entre os principais objetivos desta pesquisa, destaca-se a avaliação da ingestão de leite e substitutos e do hábito do desjejum dos universitários do curso de graduação de Nutrição da Universidade Federal do Triângulo Mineiro (UFTM), Uberaba (Minas Gerais).

\section{METODOLOGIA}

A Universidade Federal do Triângulo Mineiro (UFTM) localiza-se na cidade de Uberaba (Minas Gerais). Fundou-se no ano de 1953 com o curso de medicina e, atualmente, possui 24 cursos de graduação e, dentre eles, cursos na área da saúde (medicina, biomedicina, enfermagem, nutrição, fisioterapia, psicologia, serviço social e terapia ocupacional). No curso de graduação em nutrição há, aproximadamente, 185 alunos matriculados.
A população do estudo foi constituída por estudantes do curso de nutrição da UFTM e os critérios de inclusão para os participantes da pesquisa foram: serem estudantes regulares do primeiro ao último semestre do curso de graduação em Nutrição, estarem presentes no dia da coleta de dados, aceitarem ser voluntários e concordar, assinando o Termo de Consentimento Livre e Esclarecido.

O presente estudo é do tipo transversal e os dados foram coletados por meio da adoção do Questionário Semi-Quantitatico de Frequência Alimentar (QSFQ). O local de aplicação dos questionários foi nas salas de aula da UFTM, após a permissão do professor, ou em horários agendados previamente com os alunos.

O levantamento constou de duas partes, sendo a primeira com questões sobre a frequência de consumo ou ingestão (diária, semanal, quinzenal, mensal, nunca ou raramente) em medidas caseiras; e a segunda identificava a frequência, hábito e a ingestão no desjejum, percepção qualitativa do desjejum antes e após o ingresso na Universidade. Como variáveis demográficas o questionário considerava a idade e o sexo.

Os alimentos considerados substitutos do leite foram os queijos (minas, prato e mussarela), iogurtes, coalhada, requeijão e extrato de leite de soja fortificado com cálcio. As porções foram avaliadas conforme os intervalos de consumo, separadamente, por $0+1$ porções, $1+2$ porções, $2+3$ porções e de 3 ou mais porções e para avaliar a qualidade do desjejum pelos universitários utilizou-se os termos "muito bom", "bom", "regular", "fraco" e "ruim".

A coleta de dados foi realizada entre os meses de março a abril de 2010 pela equipe do estudo composta por quatro integrantes (alunos, pesquisador e professor) do curso de graduação em Nutrição, previamente treinados.

O estudo foi aprovado pelo Comitê de Ética em Pesquisa (CEP) UFTM contemplado pelo número 1461 do parecer, de acordo com as Diretrizes e Normas Regulamentadoras de Pesquisa envolvendo Seres Humanos, do Conselho Nacional de Saúde, Resolução n¹96, de 10 de outubro de 1996. O estudo é parte integrante do projeto intitulado "Avaliação Nutricional de Estudantes Universitários”. 
Para a análise dos dados descritivos foi realizada frequência de distribuição no programa Microsoft Office Excel 2007. Os dados sobre o consumo de leite e substitutos também passaram por processo de transformação em porções alimentares pela utilização da Pirâmide Alimentar Adaptada [ $[$ ] o qual utiliza como parâmetro de recomendação a ingestão de 3 porções diárias de leite e substitutos para atingir a recomendação diária do mineral cálcio de 1.000 mg/dia, segundo a Dietary Reference Intakes [23] para a população de estudo.

\section{RESULTADOS}

A população estudada foi composta por 111 universitários, distribuídos em diferentes etapas do curso de Graduação em Nutrição na UFTM, constando a maior participação dos estudantes do terceiro semestre $23(20,7 \%)$ e menor envolvimento dos estudantes do quarto semestre 07 (6,3\%) (Tabela 1).

Tabela 1. Distribuição da amostra de estudantes (voluntários) do Curso de Nutrição da Universidade Federal do Triângulo Mineiro, de acordo com os respectivos semestres

\begin{tabular}{cccccccccc}
\hline & & \multicolumn{9}{c}{ Semestre } & & & \\
\hline & Primeiro & Segundo & Terceiro & Quarto & Quinto & Sexto & Sétimo & Oitavo & Total \\
\hline Percentagem & 17,1 & 11,7 & 20,7 & 6,3 & 11,7 & 13,5 & 10 & 9 & 100 \\
\hline
\end{tabular}

Foram excluídos, na apuração dos dados, dez questionários e respostas dos estudantes por omitirem determinadas informações ou não completaram as questões.
Os estudantes possuíam a média de 21 anos de idade (17 a 26 anos) e foi encontrado predomínio do sexo feminino (Tabela 2).

Tabela 2. Distribuição dos estudantes do curso de Graduação em Nutrição da Universidade Federal do Triângulo Mineiro, de acordo com o gênero e semestre

\begin{tabular}{|c|c|c|c|c|c|}
\hline \multirow[t]{3}{*}{ Semestre } & \multicolumn{4}{|c|}{ Sexos } & \multirow{3}{*}{$\begin{array}{c}\text { Total } \\
\text { (n) }\end{array}$} \\
\hline & \multicolumn{2}{|c|}{ Feminino } & \multicolumn{2}{|c|}{ Masculino } & \\
\hline & $\mathrm{n}$ & $\%$ & $\mathrm{n}$ & $\%$ & \\
\hline 10 & 19 & 100,0 & 0 & 0,0 & 19 \\
\hline $2 \mathrm{o}$ & 10 & 76,9 & 3 & 23,1 & 13 \\
\hline 3o & 22 & 95,6 & 1 & 4,4 & 23 \\
\hline 4음 & 07 & 100,0 & 0 & 0,0 & 07 \\
\hline 5ㅇ & 12 & 92,3 & 1 & 7,7 & 13 \\
\hline 6o & 14 & 93,3 & 1 & 6,7 & 15 \\
\hline 7을 & 11 & 100,0 & 0 & 0,0 & 11 \\
\hline 8o & 09 & 90,0 & 1 & 0,0 & 10 \\
\hline Total & 104 & 93,7 & 7 & 6,3 & 111 \\
\hline
\end{tabular}

$\mathrm{n}=$ número de estudante $\%$ = porcentagem

A Tabela 3 demonstra o consumo das porções de leite e substitutos para os vários semestres do curso de Graduação em Nutrição. 
Tabela 3. Distribuição do consumo diário de porções de leite e substitutos dos universitários do curso de Nutrição, de acordo com os semestres, da Universidade Federal do Triângulo Mineiro

\begin{tabular}{|c|c|c|c|c|c|c|c|c|c|}
\hline \multirow[t]{3}{*}{ Semestre } & \multicolumn{8}{|c|}{ Porção } & \multirow{3}{*}{$\begin{array}{c}\text { Total } \\
\text { (n) }\end{array}$} \\
\hline & \multicolumn{2}{|c|}{$0 \vdash 1$} & \multicolumn{2}{|c|}{$1 \vdash 2$} & \multicolumn{2}{|c|}{$2+3$} & \multicolumn{2}{|c|}{3 ou mais } & \\
\hline & $\mathbf{n}$ & $\%$ & $\mathbf{n}$ & $\%$ & $\mathrm{n}$ & $\%$ & $n$ & $\%$ & \\
\hline 1 응 & 1 & 5,3 & 6 & 31,6 & 4 & 21,0 & 8 & 42,1 & 19 \\
\hline 2 은 & 2 & 15,0 & 4 & 31,0 & 3 & 23,0 & 4 & 31,0 & 13 \\
\hline 3응 & 4 & 17,4 & 5 & 21,7 & 6 & 26,0 & 8 & 34,9 & 23 \\
\hline 4o & 1 & 14,3 & 2 & 28,6 & 3 & 42,8 & 1 & 14,3 & 07 \\
\hline 5음 & 2 & 15,4 & 2 & 15,4 & 4 & 30,7 & 5 & 38,5 & 13 \\
\hline 6o & 1 & 6,6 & 4 & 26,7 & 3 & 20,0 & 7 & 46,7 & 15 \\
\hline 7으 & 0 & 0,0 & 4 & 36,3 & 3 & 27,4 & 4 & 36,3 & 11 \\
\hline 8을 & 1 & 10,0 & 2 & 20,0 & 3 & 30,0 & 4 & 40,0 & 10 \\
\hline Total & 12 & 10,8 & 29 & 26,1 & 29 & 26,1 & 41 & 37 & 111 \\
\hline
\end{tabular}

$\mathrm{n}=$ número de estudante; $\%=$ porcentagem

Desta forma, identificou-se que $37 \%$ dos estudantes ingerem de 3 ou mais porções de leite e/ou substitutos diariamente, sendo que $26,1 \%$ possuem o hábito de ingerir alimentos fontes de cálcio de 1 - 2 e de $2+3$ porções diárias e, somente, $10,8 \%$ os ingerem de 0 - 1 porções. Os períodos de maior frequência de consumo de leite e substitutos de 3 - ou mais porções por dia foram o $6^{\circ}$ período $(46,7 \%), 1^{\circ}$ período $(42,1 \%), 8^{\circ}$ período $(40,0 \%)$ e o $5^{\circ}$ período $(38,5 \%)$.
Em relação à distribuição do consumo e hábito do desjejum ao longo dos dias da semana, observou-se que $5,4 \%$ o faziam entre 1 a 3 dias na semana, $34,23 \%$ o faziam entre 4 a 6 dias na semana, mas grande parcela $(60,36 \%)$ o fazia diariamente.

$\mathrm{Na}$ Figura 1, encontra-se a relação da autoavaliação do desjejum realizada pelos estudantes.

Figura 1. Descrição da avaliação sobre a qualidade nutricional do desjejum entre os universitários do curso de Nutrição da Universidade Federal do Triângulo Mineiro

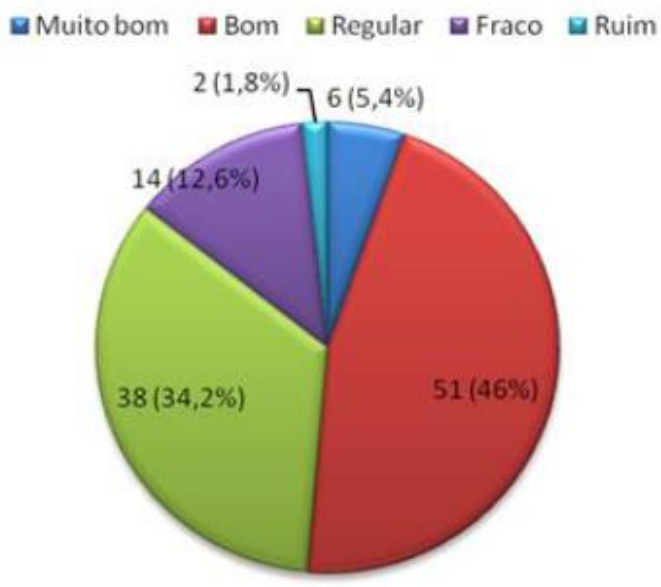

Verificou-se, na Figura 1, que 5,4\% dos estudantes consideraram o seu desjejum "muito bom", $46 \%$ classificaram como "bom", 34,2\% como "regular", 12,6\% como fraco e, 1,8\% como "ruim". A maior parte dos dados revelou que a população estudada considerava o seu desjejum como "muito 
bom" ou "bom", justificado pelo consumo de variedades de alimentos, como aqueles fontes de carboidratos, fontes de cálcio e frutas. O desjejum era classificado como "fraco" ou "ruim" quando a qualidade, quantidade e diversidade alimentar eram consideradas limitadas pelos estudantes.

Ao relacionar o hábito do desjejum com o ingresso na Universidade, 53,1\% dos estudantes referiram que houve modificações no hábito do desjejum após ingressar na Universidade e 46,9\% responderam não ter ocorrido alterações após o ingresso na instituição.

$\mathrm{Na}$ análise qualitativa realizada pelos estudantes sobre as alterações do desjejum após o ingresso na Universidade, dentre aqueles que identificaram que houveram modificações no desjejum após o ingresso na Universidade, $54,2 \%$ consideraram que as mudanças foram para "melhor" e 45,8\% para "pior".

\section{DISCUSSÃO}

Considera-se que a qualidade de vida se expressa nas alterações do comportamento alimentar e, desta forma, é de grande importância avaliar de forma qualitativa e quantitativa o consumo e ingestão de alimentos e nutrientes para promover tais modificações benéficas ${ }^{[4]}$.

O leite e seus substitutos como queijos, requeijão, iogurte e coalhada são considerados os alimentos principais fontes de cálcio, desta forma, quando consumidos em porções recomendadas atinge-se o valor de referência diário do mineral [5]. Neste estudo, a ingestão de três ou mais porções do grupo dos alimentos lácteos, preconizado como referência para atendimento às recomendações diárias foi relativamente elevado $(37 \%)$.

No estudo de Monteiro et al. [24], o consumo de leite e queijo foi encontrado em $91 \%$ e $49 \%$ entre as discentes de nutrição e enfermagem, respectivamente, com idade entre 19 a 27 anos, de uma Universidade Pública brasileira.

Em estudo realizado Durá Travé [25], para verificar o consumo de leite e derivados em universitários ( $\mathrm{n}=500$ ), constatou-se que $98,6 \%$ da população de estudo referia ter ingerido algum produto lácteo nas últimas 24 horas. Este dado era expresso pelo recordatório de 24 horas e o leite estava presente em $92 \%$ deles, seguido por $60 \%$ do iogurte, $32,6 \%$ do queijo e dos $13 \%$ distribuídos em outras fontes, sendo que o maior consumo destes alimentos foi no desjejum.

No estudo de Larson et al. [19] os autores obtiveram a média do consumo de porções de leite de 2,9 para os meninos e 2,4 porções para as meninas de ensino médio $(n=4.746)$ e, neste mesmo estudo, os autores destacaram a importância de intervenções com foco no ambiente familiar já que são mais eficazes no aumento da ingestão de cálcio entre os adolescentes.

O leite e seus substitutos são fontes principais do mineral cálcio e, existindo inadequação deste nutriente no período de adolescência e na fase adulta, acarretam-se consequências, como surgimento de osteoporose pela ingestão insuficiente do cálcio [26].

O hábito do desjejum junto a uma alimentação adequada está relacionado com a promoção e melhorias no desenvolvimento fisiológico e do conhecimento [10]. Desta forma, neste estudo, observou-se que o hábito do desjejum estava presente em grande parcela da população $(60,36 \%)$ que o realizavam diariamente, mostrando comportamento benéfico à saúde.

Semelhante expressão dos dados deste trabalho também foi encontrada em estudos onde $86 \%$ dos adolescentes que tinham o hábito do desjejum [27]. Em outro estudo, foram encontrados dados positivos quanto à realização do desjejum diariamente na maioria $(n=116)$ dos adolescentes de ambos os sexos, estudantes de escolas particulares, referentes ao total da amostra de 214 alunos [16].

Molinero et al. [28] revelam um estudo com 738 alunos desde a escola primária até o ensino médio, de ambos os sexos, o qual grande parte dos alunos $(59,1 \%)$ realizavam o desjejum todos os dias da semana e apenas $9,6 \%$ o realizava entre 4 a 6 dias da semana.

Um estudo realizado com 232 estudantes do primeiro e último semestre de cursos da área da saúde de uma universidade brasileira revelou que considerável parcela da população estudada $(73,3 \%)$ fazia o desjejum de cinco a sete vezes durante a semana e 59,5\% faziam o desjejum diariamente ${ }^{[10]}$. 
Segundo estudo realizado por Durá Travé [29] com 400 estudantes do ensino médio de uma escola pública rural na Espanha, 93,2\% dos estudantes faziam o desjejum, sendo que, $89,3 \%$ deles ingeriam leite, como também, no estudo de Rivas et al. [30] contatou-se que do total de 402 adolescentes, 95\% deles realizavam o desjejum, o qual, o leite foi um dos alimentos mais consumidos (47\%).

A relação de indivíduos que possuem o hábito regular do desjejum com as vantagens nutricionais é positiva, sendo estas: redução do consumo de alimentos ricos em lipídeos ao longo do dia, redução do consumo de lanches não nutritivos "snacks", influenciando no aumento da ingestão de nutrientes essenciais e de baixa caloria, associando-se às alterações do peso corporal [31].

O consumo de alimentos nutritivos no desjejum faz com que o organismo responda melhor na realização das atividades durante o dia e, desta forma, é considerado uma refeição importante e, principalmente, quando conciliada à ingestão de leite $\mathrm{e}$ substitutos para suprir as necessidades de cálcio ${ }^{[10] .}$

Quanto à avaliação perceptiva dos estudantes, o presente estudo observou que a maioria $(46 \%)$ avaliou o desjejum como "Bom" e 38\% escolheram a opção "Regular". Demais percentuais foram alocados, respectivamente em ordem decrescente, como "Fraco", "Muito bom" e "Ruim".

Já em estudo de Lozano e Ballesteros [32] em que $60,3 \%$ classificaram que a qualidade do desjejum era passível de melhorias já que falta um dos grupos alimentares, sendo estes lácteos, cereais e frutas.

De acordo com o estudo de Pinheiro [10], os integrantes do curso de Nutrição, dentre outros da área da saúde, foram aqueles que, ao avaliarem o desjejum, reconheceram a grande relevância dos mesmos. Tal opinião deve-se, possivelmente, ao estudo mais aprofundado sobre a nutrição e a alimentação quando comparados aos alunos de outros cursos.

No estudo com 105 alunos com cursos da área da saúde observou-se que sobre os conhecimentos sobre a alimentação e composição nutricional, os alunos do curso de Nutrição demonstraram possuir maiores informações aos outros cursos, porém, quanto ao hábito alimentar e o estilo de vida dos estudantes, os cursos apresentaram- se semelhantes e que $78-90 \%$ dos estudantes realizavam o desjejum diariamente e $37 \%$ variavam nos finais de semana [33].

Roberto e Carlos [34] estudaram 243 alunos, de ambos os sexos, os quais foram separados em grupo controle e grupo intervenção. O grupo intervenção recebeu orientações pelo programa de educação nutricional no início e no final do curso escolar. Constatou-se que os alunos do grupo intervenção obtiveram melhorias na qualidade do desjejum (de $23 \%$ para $32,6 \%$ e havia redução do consumo de alimentos de má qualidade (de 4,4\% para 3,7\%), demonstrando resultados positivos da intervenção de educação nutricional na ajuda da melhoraria na qualidade da refeição. Estes dados podem ser relacionados com o presente estudo, cujos alunos do curso de nutrição recebem tais orientações no decorrer do bacharelado.

As consequências do desempenho mental, cognição e aprendizagem, e do equilíbrio nutricional estão relacionadas com os benefícios do desjejum na idade escolar, aumentando a frequência escolar e, na vida adulta e na velhice, melhorando a qualidade das dietas $[35,36,37]$.

As alterações no humor foram relatadas por $51,3 \%$ dos estudantes universitários do presente estudo o que expressa a maior parte da população e as alterações mais referidas quando o desjejum era omitido foi o mau-humor $(26,4 \%)$ e a irritação $(25 \%)$. A omissão da refeição do desjejum pode provocar sintomas desagradáveis no organismo, como, dor de cabeça, fome, fraqueza e tontura aumentando a chance de mau-humor ${ }^{[38]}$.

Este estudo revela que 53,1\% dos estudantes universitários do curso de Nutrição consideraram ter tido modificações alimentares após ingressar na Universidade e 46,9\% desconsideraram tal alteração. Dentre os que avaliaram qualitativamente as alterações, grande parcela $(54,2 \%)$ referiu que as modificações foram para "melhor" e 45,8\% para "pior".

Desta forma, presume-se que o curso de Graduação em Nutrição influenciaria no consumo e ingestão alimentar no desjejum dos estudantes que responderam que as alterações foram benéficas. Para aqueles estudantes que responderam "pior" (45,8\%) parece que a vida universitária e seus inúmeros compromissos torna a "falta de tempo" para se 
alimentar no período da manhã um grande problema alimentar.

Vieira [3] demonstra em seu estudo que 50\% dos estudantes entrevistados relataram ingerir maior quantidade de alimentos após o ingresso na universidade e Alves e Boog [1] justifica a redução da qualidade alimentar pelas limitações que ocorrem na vida universitária, considerando ser uma experiência nova e que demanda tempo e dedicação, paralelos, às atividades acadêmicas.

\section{CONCLUSÃO}

Pôde-se notar que cursar a Graduação em Nutrição pode influenciar no consumo do leite e seus substitutos (fontes de cálcio), além dos hábitos alimentares do desjejum. Parece que as informações nutricionais durante o curso destacam a importância da alimentação saudável para prevenção de agravos à saúde, favorecendo a mudança positiva dos hábitos.

O consumo de leite e substitutos e o hábito do desjejum dos estudantes, por mais que tenham sido positivos, ainda necessitam de melhorias para que a população obtenha o mineral cálcio pelo leite e pelos produtos lácteos, através das práticas alimentares e suprimento alimentar.

O hábito do desjejum deve ser incentivado pela importância diária, independente das tarefas, pois seu benefício é de ampla significância fisiológica, principalmente, incluindo alimentos saudáveis e o leite e seus substitutos.

Análises que envolvam os estudantes, mesmo àqueles vinculados a informação nutricional, tornamse valiosas por fazerem parte da classe de futuros multiplicadores da área da saúde.

\section{REFERÊNCIAS}

[1] Alves HJ, Boog MCF. Comportamento alimentar em moradia estudantil: um espaço para promoção da saúde. Rev Saúde Pública. 2007;41(2):197-204.

[2] Niemeier HM, Raynor HA, Lloyd-Richardson EE, Rogers ML, Wing RR. Fast food consumption and breakfast skipping: predictors of weight gain from adolescence to adulthood in a Nationally Representative Sample. J Adolesc Health. 2006;39:842-849.
[3] Vieira VCR, Priore SE, Ribeiro SMR, Franceschini SCC, Almeida LP. Perfil socioeconômico, nutricional e de saúde de adolescestes recém-ingressos em uma universidade pública brasileira. Rev Nutr. 2002;15(3):273-282.

[4] Marchioni DML, Slater B, Fisberg RM. Aplicação das Dietary Reference Intakes na avaliação de ingestão de nutrientes para indivíduos. Rev Nutr. 2004;17(2):207-216.

[5] Pereira GAP, Genaro PS, Pinheiro MM, Szejnfeld VL, Martini LA. Cálcio dietético - estratégias para otimizar o consumo. Rev. Bras. Reumatol. 2009;49(2):164-80.

[6] Philippi ST, Latterza AR, Cruz ATR, Ribeiro LC. Pirâmide Alimentar Adaptada: Guia para escolhas dos alimentos. Rev Nutr. 1999;12(1):65-80.

[7] Porto F. Análise de ingestão de cálcio de adolescentes de São Leopoldo [tese de doutorado]. Porto Alegre: Pontífica Universidade Católica do Rio Grande do Sul; 2005.

[8] Galeazzi MAM, Domene SMA, Schieri R. (org.). Estudo multicêntrico sobre consumo alimentar. Rio de Janeiro: Ministério da Saúde, 1997.

[9] Anderson JJB. Nutrição e saúde óssea. In: Mahan KL, Escott-Stump S. Krause, alimentos, nutrição \& dietoterapia. 11. ed. São Paulo: Roca; 2005.

[10] Pinheiro KAPN. Perfil do consumo de desjejum de estudantes da Área de Saúde da Universidade de Brasília, DF [tese de doutorado]. Brasília: Universidade de Brasilia; 2006.

[11] Gambardella AMD, Frutuoso MFP, Franch C. Prática alimentar de adolescentes. Rev Nutr. 1999;12(1):5-19.

[12] Acosta MM. Influencia del desayuno sobre da función cognocitiva de escolares de uma zona urbana de Valencia, Venezuela. Arch Latinoam Nutr. 2001;51(1):57-63.

[13] Rosenburg O. O desjejum dos alunos das quatro principais primeiras séries de nível I das escolas da rede municipal de ensino de São Paulo. Rev Saúde Pública. 1977;11(4).

[14] Silva MV. Alimentos consumidos pelos alunos de escolas de tempo integral. Alimen. Nutr. 2000;11:89-110.

[15] Lerner BR, Lei DLM, Chaves SP, Freire RD. O cálcio consumido por adolescentes de escolas públicas de Osasco, São Paulo. Rev Nutr. 2000;13(1):57-63.

[16] Silva HHC. Consumo alimentar do desjejum de adolescentes em escolas particulares de Brasília, DF [tese de doutorado]. Brasilia: Universidade de Brasília; 2006. 
[17] Matthys C, Henauw S, Bellemans M, Maeyer M, Backer G. Breakfast habits affect overall nutrient profiles in adolescents. Public Health Nutrition. 2006;10(4):413-21.

[18] Marcondelli P, Costa THM, Schmitz BAS. Nível de atividade física e hábitos alimentares de universitários do $3^{\circ}$ ao $5^{\circ}$ semestre da área da saúde. Rev Nutr. 2008;21(1):39-47.

[19] Larson NI, Story M, Wall M, Neumark-Sztainer D. Calcium and dairy intakes of adolescents are associated with their home environment, taste preferences, personal health beliefs, and meal patterns. J Am Diet Assoc. 2006;106(11):1816-24.

[20] Vieira VCR, Priore SE, Ribeiro SMR, Franceschini SCC. Alterações no padrão alimentar de adolescentes com adequação pôndero-estatural e elevado percentual de gordura corporal. Rev. Bras. Saúde Mater. Infant. 2005;5(1):93-102.

[21] Costa MCD, Cordoni Júnior L, Matsuo, T. Hábito alimentar de escolares adolescentes de um município do oeste do Paraná. Rev Nutr. 2007;20(5):461-471.

[22] Garcia GCB, Gambardella AMD, Frutuoso MFP. Estado nutricional e consumo alimentar de adolescentes de um centro de juventude da cidade de São Paulo. Rev Nutr. 2003;16(1):41-50.

[23] DRI. Food and Nutrition Board, Institute of Medicine. Dietary reference intakes for calcium, phosphorus, mgnesium, vitamin D, and fluoride. Washington, DC: National Academy Press; 1997.

[24] Monteiro MRP, Andrade MLO, Zanirati VF, Silva RR. Hábito e consumo alimentar de estudantes do sexo feminino dos cursos de enfermagem de uma universidade pública. Rev APS. 2009;12(3):271-277.

[25] Durá Travé T. Ingesta de leche y derivados lácteos em la población universitária. Nutr Hosp. 2008;23(2):89-94.

[26] Saito MI. Nutrição. In: Marcondes E. Pediatria Básica. 8. ed. São Paulo: Sarvier; 1999. p. 555-560.

[27] Rego Filho EA, Vier BP, Campos E, Günther LA, Carolino IR. Avaliação nutricional de um grupo de adolescentes. Acta Sci. Health Sci. 2005;27(1):63-67.
[28] Molinero O, Piñero-Castro J, Ruiz JR, González Montesinos JL, Mora J, Márquez S. Conductas de salud em escolares de la província de Cádiz. Nutr Hosp. 2010;25(2):280-289.

[29] Durá Travé T. El desayuno de los alumnos de Educación Secundaria Obligatoria (ESO). Nutr Hosp. 2002;17(4):189-196.

[30] Rivas, P. R.; Figueredo, C. R.; Lanza, T. A.; GonzálezLamuño, D, Fuentes MG. Desayuno y almuerzo de los adolescentes escolarizados de Santander. Nutr Hosp. 2005;10(3):217-222.

[31] Schlundt DG, Hill JO, Sbrocco T, Pope-Cordle J, Sharp $\mathrm{T}$. The role of breakfast in the treatment of obesity: a randomized clinical trial. Am J Clin Nutr. 1992;55:645-651.

[32] Lozano RH, Ballesteros JCF. Estudio sobre el desayuno y el rendimiento escolar em um grupo de adolescentes. Nutr Hosp. 2006;21(3):346-352.

[33] Bravo AM, Martín NÚ, González AG. Evaluación de los hábitos alimentarios de una población de estudiantes universitarios en relación con sus conocimientos nutricionales. Nutr Hosp. 2006;21(4):466-73.

[34] Roberto HL, Carlos FBJ. Influencia de un programa de educación nutricional en la modificación del desayuno en un grupo de adolescentes. Nutr. Clín. Diet. Hosp. 2010;30(2):26-32.

[35] Hultman E. Nutritional effects on work performance. Am J Clin Nutr. 1989;49:949-957.

[36] Michaud C, Musse N, Nicolas JP, Mejean L. Effects of breakfast-size on hort-tern memory concentration, mood and blood glucose. J Adolesc Health. 1991;12(1):53-57.

[37] Pollitt E, Mathews R. Breakfast and cognition: an integrative summary. Am J Clin Nutr. 1988;67:804-813.

[38] Bispo JS. Perfil alimentar referente ao desjejum dos professores da universidade de Brasília [dissertação de mestrado]. Brasília: Universidade de Brasília; 2006. 